\title{
Prognostic value of site-specific metastases and therapeutic roles of surgery for patients with metastatic bladder cancer: a population-based study
}

This article was published in the following Dove Press journal:

Cancer Management and Research

14 November 2017

Number of times this article has been viewed

Background: We aimed to evaluate the prognostic value of site-specific metastases in patients with metastatic bladder cancer and analyze the roles that surgeries play in the treatment of this malignancy.

Materials and methods: A population-based retrospective study using Surveillance, Epidemiology and End Results dataset was performed and metastatic bladder cancer patients were classified according to the sites of metastases (bone, brain, liver, lung and distant lymph nodes). Kaplan-Meier analysis with log-rank test was used for survival comparisons. Multivariate Cox regression model was employed to analyze the effect of distant metastatic sites on overall survival (OS) and cancer-specific survival (CSS).

Results: A total of 1862 patients with metastatic bladder cancer from 2010 to 2014 were identified. Bone, lung and distant lymph nodes were the most common metastatic sites. Patients with bone, brain, liver and lung involvement had worse OS and CSS compared to patients without the corresponding sites of metastases. Multivariate analysis showed that bone, brain, liver and lung metastases were independent prognostic factors for both OS and CSS, while distant node metastasis was not. Moreover, patients with a single metastatic site had more favorable OS $(p<0.001)$ and CSS $(p<0.001)$ than patients with multisite metastases. Among single-site metastatic patients, distant nodes and liver metastases represented the best and the worst prognosis, respectively. Moreover, radical cystectomy was an independent predictor for better OS and CSS, while in patients with liver metastasis and multiple metastatic sites, RC did not bring benefits. Besides, in patients with a single metastatic site, metastasectomy seemed to be associated with favorable OS ( $p=0.042)$, especially for patients with age $<65$ years $(p=0.006)$ and for muscleinvasive bladder cancer patients $(p=0.031)$.

Conclusion: Distant metastatic sites have differential impact on survival outcomes in patients with metastatic bladder cancer. Surgeries, including radical cystectomy and metastasectomy, might still lead to survival benefits for highly selected patients.

Keywords: bladder cancer, metastatic site, prognosis, radical cystectomy, metastasectomy, Surveillance, Epidemiology and End Results

\section{Introduction}

Bladder cancer ( $\mathrm{BCa}$ ) ranks number seven in the list of the most common malignancies in male patients, ${ }^{1}$ with over 79,000 new cases and over 16,000 deaths a year reported in the USA. ${ }^{2}$ Among all urinary tumors, the morbidity and mortality of BCa were both the second highest, ${ }^{3}$ and the disease is not easy to cure due to the high recurrence and metastasis rates, with a 5 -year survival rate $\sim 75 \% .{ }^{4}$ Despite the majority of

Correspondence: Zhoujun Shen Hospital, Fudan University, No. 12 Middle Urumqi Road, Shanghai 200040, China

Tel +86 2l 52888228

Fax +862162485237

Email shenzj68@sina.cn 
patients having non-muscle-invasive $\mathrm{BCa}$, the disease might easily invade the muscle layer, which leads to a significantly worse prognosis. According to the European Association of Urology guidelines 2017 of $\mathrm{BCa}, 50 \%$ of muscle-invasive $\mathrm{BCa}$ (MIBC) relapsed after radical cystectomy (RC) and distant metastases are more common compared to local recurrence. ${ }^{5}$ Also, what is more unfortunate is that $10 \%-15 \%$ of BCa patients are already metastatic at diagnosis. ${ }^{6}$

In the setting of metastatic $\mathrm{BCa}$, distant lymph nodes and lung are the most come sites of metastases, while the proportion of bone metastasis remains controversial. ${ }^{7,8}$ Some cases could undergo a change of the metastatic pattern and involve other distant organs. Patients with different metastatic sites might represent different subgroups of patients with different tumor biologic patterns and prognosis and subsequently, distinct therapeutic approaches. ${ }^{7,9}$ Previous studies had pointed out that liver metastases might be a predictor for poor prognosis in metastatic urothelial carcinoma patients. ${ }^{10-12}$ Nevertheless, due to the limited number of patients and differences in treatment strategies, the effects of bone metastases, distant lymph node metastases as well as different sorts of visceral metastases on the prognosis of metastatic BCa patients remain blurry.

The up-to-date guidelines still recommend chemotherapy as the first-line treatment of metastatic $\mathrm{BCa} .{ }^{5}$ In spite of a passable initial response rate to chemotherapy, the median overall survival of the disease was still unsatisfactory. ${ }^{13}$ Since no huge progress has been made in effective chemotherapy, patients with metastatic BCa barely had a median survival that surpassed 3-6 months. ${ }^{14}$ This calls for the reconsideration of the current therapeutic strategies for this disease. Previous studies suggested that benefits of long-term cancer control from metastasectomy might be obtained in highly selected patients, ${ }^{8}$ especially in those with lung metastases. ${ }^{15,16}$ However, previous studies were all based on rather small number of single-institutional patient cohorts; thus, the roles that surgeries, including surgeries of the primary tumor and metastasectomy, played in the treatment of metastatic BCa are still disputable.

Population-based analysis on the prognostic value of site-specific metastases and $\mathrm{RC}$ as well as metastasectomy for metastatic $\mathrm{BCa}$ is deficient. Therefore, based on a large cohort of patients, the purpose of this study is to investigate the prognostic value of site-specific metastases. We also analyzed the effect of RC and metastasectomy on metastatic $\mathrm{BCa}$ in the hope of offering reliable evidence for the management of metastatic BCa.

\section{Materials and methods}

\section{Study population}

Patients diagnosed with metastatic (M1 stage) BCa from 2010 to 2014 were identified from Surveillance, Epidemiology and End Results (SEER) database because the detailed information about distant metastatic sites before 2010 was not available. Other inclusion criteria were as follows: patients $>16$ years old; $\mathrm{BCa}$ as the primary cancer diagnosis; distant metastatic sites including bone, brain, liver, lung and distant lymph nodes; active follow-up and patients with $>0$ day of survival. Patients without sufficient information about distant metastatic sites or survival data were excluded. We also excluded patients with controversial information (e.g., patients at N1-N3 stages with 0 positive lymph node).

\section{Covariates and follow-up information}

Covariates of interest extracted for each case included age at diagnosis, gender, race, marital status, tumor size, American Joint Committee on Cancer eighth T stage, American Joint Committee on Cancer eighth $\mathrm{N}$ stage, site of metastases, surgery to the primary and metastases. The follow-up information including survival status, survival months and cause of death were all extracted from the dataset. The primary endpoints of the study were overall survival (OS) and cancerspecific survival (CSS). Survival time was calculated from the date of diagnosis to the date of 1) death from any cause (OS) 2) death from BCa (CSS) or 3) the last follow-up.

\section{Statistical analysis}

Chi-square test was used to compare the patient characteristics between groups. Venn diagram was drawn to illustrate the distribution of distant metastatic sites in patients. Survival curves were estimated using the Kaplan-Meier method. The log-rank test and Breslow test were used to assess significant differences for OS and CSS. Multivariate Cox proportional hazards regression analysis was employed to evaluate the prognostic factors, and hazard ratios (HR) along with $95 \%$ CI were calculated. Two-sided $p$ values of $<0.05$ were considered statistically significant. Venn diagram was drawn using R version 3.4.0 (http://www.r-project.org/). Other analyses were performed using the SPSS version 22.0 software package (IBM Corporation, Armonk, NY, USA).

\section{Compliance with ethical standards}

All procedures performed in studies involving human participants were in accordance with the ethical standards of the institutional and national research committee and with 
the 1964 Helsinki Declaration and its later amendments or comparable ethical standards. We obtained permission to access SEER dataset with the reference number 11587 Nov2016. Extraction of data from the SEER database does not require informed consent.

\section{Results}

\section{Patients' characteristics}

A total of 1862 patients with M1 stage BCa were identified in the period from 2010 to 2014 and included in our analysis according to the inclusion and exclusion criteria. The characteristics of all patients are presented in Table 1, and the distribution of patients in each year (from 2010 to 2014) was roughly even. The median age of all patients was 70 (range 17-98). The majority of the cohort (85.3\%) was ethnically white, and 1336 (71.8\%) patients were male patients. As for the tumor stage and grade, $1250(67.1 \%)$ patients were suffering from MIBC and 1379 (74.1\%) patients had G3 tumor. Only 124 (6.7\%) and 95 (5.1\%) patients received RC and metastasectomy, respectively. The follow-up time ranged from 0 to 59 months, and 1558 $(83.7 \%)$ patients had died before the last follow-up, of which $1426(76.6 \%)$ deaths were due to BCa. Statistically significant correlations obtained between different characteristics and each site of metastases are also shown in Table 1.

\section{Distribution of distant metastatic sites}

The distribution of distant metastatic sites is summarized in Table 1, and we applied Venn diagram (Figure 1) to further illustrate metastatic sites distribution. The Venn diagrams show the number of patients with different kinds of metastatic sites. It shows that $799(42.9 \%)$ patients were diagnosed with bone metastases, 724 (38.9\%) patients were with lung metastases, 637 (34.2\%) patients were with distant lymph nodes metastases, 493 (26.5\%) patients were with lung metastases and only $76(4.1 \%)$ patients had brain metastases. A total of 1228 (66.0\%), 438 (23.5\%), $153(8.2 \%)$ and 43 (2.3\%) patients had one, two, three and four metastatic sites, respectively, and the overlapping area of the Venn diagram presents patients with multiple metastatic sites. No patient was found to have five metastatic sites in the cohort.

\section{Impact of metastatic sites on survival outcomes}

The OS and BCa specific survival were compared according to different metastatic sites. Kaplan-Meier analyses showed that patients with bone (Figure 2A, B), brain (Figure 2C, D), liver (Figure 2E, F) or lung metastases (Figure 2G, H) had worse outcomes both for OS and CSS, compared to patients without the corresponding sites of metastases (with vs without bone metastases: $p=0.005$ for OS and $p=0.002$ for CSS; with vs without brain metastases: $p<0.001$ for both OS and CSS; with vs without liver metastases: $p<0.001$ for both OS and CSS; with vs without lung metastases: $p<0.001$ for both OS and CSS). For distant lymph node involvement, however, patients with other kinds of metastases rather than distant node metastases showed worse OS $(p=0.001)$ and CSS ( $p=0.001)$, as shown in Figure 2I, J.

The effect of the number of distant metastatic sites on survival was further analyzed. Results revealed that patients with single-site metastasis had significantly better outcomes for both OS and CSS than patients with two, three or four metastatic sites (all $p<0.001$ ), as shown in Figure 3A, B. Moreover, patients with two-site metastases also showed survival advantages over "three sites" group $(p<0.001$ for OS; $p=0.004$ for CSS) and "four sites" group ( $p<0.001$ for OS; $p=0.004$ for CSS). No significant survival difference was observed between patients with three- and four-site metastases ( $p=0.522$ for OS; $p=0.385$ for CSS), and overlapping survival curves of these two groups are shown in Figure 3A (for OS) and 3B (for CSS).

In the multivariate Cox analyses for the overall cohort (Table 2), in terms of distant metastatic sites, patients with bone (OS: $\mathrm{HR}=1.679,95 \% \mathrm{CI}=1.204-2.340, p=0.002$; CSS: $\mathrm{HR}=1.659,95 \% \mathrm{CI}=1.172-2.349, p=0.004)$, brain (OS: HR=2.462, 95\% CI=1.450-4.181, $p=0.001$; CSS: $\mathrm{HR}=2.461,95 \% \mathrm{CI}=1.428-4.240, p=0.001$ ), liver (OS: $\mathrm{HR}=2.038,95 \% \mathrm{CI}=1.458-2.848, p<0.001 ; \mathrm{CSS}: \mathrm{HR}=1.935$, $95 \% \mathrm{CI}=1.362-2.747, p<0.001)$ and lung metastases (OS: $\mathrm{HR}=1.995,95 \% \mathrm{CI}=1.393-2.745, p<0.001 ; \mathrm{CSS}: \mathrm{HR}=1.887$, 95\% $\mathrm{CI}=1.323-2.693, p<0.001)$ were independent prognostic factors for both OS and BCa specific survival, whereas distant lymph node metastases were not an independent prognostic indicator $(\mathrm{OS}: \mathrm{HR}=1.262,95 \% \mathrm{CI}=0.896-1.777, p=0.183$; CSS: $\mathrm{HR}=1.207,95 \% \mathrm{CI}=0.844-1.726, p=0.304)$. Besides, multisite metastases were not an independent predictor for poor survival prognosis compared with a single-site metastasis, according to the multivariate Cox analyses (Table 2).

The survival outcomes of patients with single-site metastasis were additionally analyzed, and patients with bone-only and lung-only metastases had a better prognosis compared to patients with liver metastases (bone only vs liver only: $p=0.004$ for OS and $p=0.008$ for CSS; lung only vs liver only: $p=0.019$ for OS and $p=0.025$ for CSS) as shown in Figure 4A, B, while patients with distant nodeonly metastases were significantly more likely to have a favorable prognosis than other groups of patients. Due to the 
Table I Characteristics of patients and metastatic sites

\begin{tabular}{|c|c|c|c|c|c|c|c|}
\hline \multirow[t]{2}{*}{ Characteristics } & \multirow{2}{*}{$\begin{array}{l}\text { Total }(\mathrm{N}=1862) \\
\mathrm{n}(\%)\end{array}$} & \multicolumn{2}{|c|}{ Bone metastases } & \multirow[t]{2}{*}{$p$-Value } & \multicolumn{2}{|c|}{ Brain metastases } & \multirow[t]{2}{*}{$p$-Value } \\
\hline & & Yes (\%) & No (\%) & & Yes (\%) & No (\%) & \\
\hline \multicolumn{8}{|c|}{ Age at diagnosis, years } \\
\hline Median (range) & 70 (17-98) & $70(2 \mid-97)$ & 70 (17-98) & $<0.001$ & $70(21-96)$ & 70 (17-98) & 0.113 \\
\hline$\leq 60$ & $444(23.8)$ & $217(48.9)$ & $227(5 \mathrm{I} . \mathrm{I})$ & & $20(4.5)$ & $424(95.5)$ & \\
\hline $61-70$ & $518(27.8)$ & $237(45.8)$ & $28 I(54.2)$ & & $29(5.6)$ & $489(94.4)$ & \\
\hline $7 I-80$ & $516(27.7)$ & $208(40.3)$ & $308(59.7)$ & & $15(2.9)$ & $50 \mathrm{I}(97.1)$ & \\
\hline$>80$ & $384(20.6)$ & $137(35.7)$ & $247(64.3)$ & & $12(3.1)$ & $372(96.9)$ & \\
\hline \multicolumn{8}{|l|}{ Year of diagnosis } \\
\hline 2010 & $352(18.9)$ & $152(43.2)$ & $200(56.8)$ & 0.370 & $23(6.5)$ & $329(93.5)$ & 0.090 \\
\hline 2011 & $354(19.0)$ & $142(40.1)$ & $212(57.9)$ & & $15(4.2)$ & $339(95.8)$ & \\
\hline 2012 & $387(20.8)$ & $182(47.0)$ & $205(53.0)$ & & II (2.8) & $376(97.2)$ & \\
\hline 2013 & $376(20.2)$ & $161(42.8)$ & $215(57.2)$ & & $15(4.0)$ & $361(96.0)$ & \\
\hline 2014 & $393(21.1)^{\prime}$ & $162(4 \mid .2)$ & $23 I(58.8)$ & & $12(3.1)$ & 381 (96.9) & \\
\hline \multicolumn{8}{|l|}{ Race } \\
\hline White & $1589(85.3)$ & $680(42.8)$ & $909(57.2)$ & 0.713 & $67(4.2)$ & $1522(95.8)$ & 0.552 \\
\hline Black & $191(10.3)$ & $84(44.0)$ & $107(56.0)$ & & $5(2.6)$ & $186(97.4)$ & \\
\hline Others & $80(4.3)$ & $35(43.8)$ & $45(56.2)$ & & $4(5.0)$ & $76(95.0)$ & \\
\hline Unknown & $2(0.1)$ & $0(0)$ & $2(100 \%)$ & & $0(0)$ & $2(100 \%)$ & \\
\hline \multicolumn{8}{|l|}{ Gender } \\
\hline Male & 1336 (71.8) & $609(45.6)$ & 727 (54.4) & $<0.001$ & $53(4.0)$ & $1283(96.0)$ & 0.697 \\
\hline Female & $526(28.2)$ & $190(36.1)$ & $336(63.9)$ & & $23(4.4)$ & $503(95.6)$ & \\
\hline \multicolumn{8}{|l|}{ Marital status } \\
\hline Married & $883(47.4)$ & $373(42.2)$ & $510(57.8)$ & 0.710 & $37(4.2)$ & $846(95.8)$ & \\
\hline Unmarried & $900(48.3)$ & $389(43.2)$ & $511(56.8)$ & & $36(4.0)$ & $864(96.0)$ & \\
\hline Unknown & $79(4.3)$ & $37(46.8)$ & $42(53.2)$ & & $3(3.8)$ & $76(96.2)$ & \\
\hline \multicolumn{8}{|l|}{ Grade } \\
\hline GI & $17(0.9)$ & $6(35.3)$ & II (64.7) & 0.097 & $0(0)$ & $17(100)$ & 0.222 \\
\hline $\mathrm{G} 2$ & $69(3.7)$ & $26(37.7)$ & $43(62.3)$ & & $4(5.8)$ & $65(94.2)$ & \\
\hline G3 & $1379(74.1)$ & $576(41.8)$ & $803(58.2)$ & & $50(3.6)$ & $1329(96.4)$ & \\
\hline Unknown & $397(21.3)$ & $191(48.1)$ & $206(51.8)$ & & $22(5.5)$ & $375(94.5)$ & \\
\hline \multicolumn{8}{|l|}{ T stage } \\
\hline TI & $309(16.6)$ & $124(40.1)$ & 185 (59.9) & 0.793 & $10(3.2)$ & $299(96.8)$ & 0.036 \\
\hline $\mathrm{T} 2$ & 765 (4I.I) & $331(43.3)$ & $434(56.7)$ & & $30(4.0)$ & $735(96.0)$ & \\
\hline T3 & I $58(8.5)$ & $67(42.4)$ & $91(57.6)$ & & $5(3.2)$ & $153(96.8)$ & \\
\hline $\mathrm{T} 4$ & $327(17.6)$ & $140(42.8)$ & $187(57.2)$ & & $9(2.8)$ & $318(97.2)$ & \\
\hline$T x$ & $303(16.3)$ & $137(45.2)$ & $166(54.8)$ & & $22(7.3)$ & 281 (92.7) & \\
\hline \multicolumn{8}{|l|}{$\mathrm{N}$ stage } \\
\hline No & $955(51.3)$ & 437 (45.8) & $518(54.2)$ & 0.005 & $44(4.6)$ & 911 (95.4) & 0.237 \\
\hline $\mathrm{NI}$ & $197(10.6)$ & $73(37.1)$ & $124(62.9)$ & & $6(3.0)$ & $191(97.0)$ & \\
\hline N2 & $353(19.0)$ & $135(38.2)$ & $218(61.8)$ & & $8(2.3)$ & 345 (97.7) & \\
\hline N3 & $124(6.7)$ & $43(34.7)$ & $81(65.3)$ & & $5(4.0)$ & $119(96.0)$ & \\
\hline $\mathrm{Nx}$ & $233(12.5)$ & $\mathrm{III}(47.6)$ & $122(52.4)$ & & $13(5.6)$ & $220(94.4)$ & \\
\hline \multicolumn{8}{|l|}{ Tumor size, $\mathrm{cm}$} \\
\hline$\leq 2$ & $55(3.0)$ & $21(38.2)$ & $34(61.8)$ & 0.010 & $\mathrm{I}(\mathrm{I} .8)$ & $54(98.2)$ & 0.584 \\
\hline $2-5$ & $404(21.7)$ & 161 (39.9) & $243(60.1)$ & & $17(4.2)$ & $387(95.8)$ & \\
\hline$>5$ & $430(23.1)$ & $160(37.2)$ & $264(61.4)$ & & $14(3.3)$ & $416(96.7)$ & \\
\hline Unknown & $973(52.3)$ & $45 \mathrm{I}(46.4)$ & $522(53.6)$ & & $44(4.5)$ & $929(95.5)$ & \\
\hline \multicolumn{8}{|l|}{ Radical cystectomy } \\
\hline Yes & $124(6.7)$ & $38(30.6)$ & $86(69.4)$ & 0.005 & $3(2.4)$ & $12 \mid(97.6)$ & 0.369 \\
\hline No & $1738(93.3)$ & $761(43.8)$ & $977(56.2)$ & & $73(4.2)$ & $1665(95.8)$ & \\
\hline \multicolumn{8}{|l|}{ Metastasectomy } \\
\hline Yes & $95(5.1)$ & $35(36.8)$ & $60(63.2)$ & 0.242 & $12(12.6)$ & $83(87.4)$ & $<0.001$ \\
\hline No & $1767(94.9)$ & $764(43.2)$ & $1003(56.8)$ & & $64(3.6)$ & $1703(96.4)$ & \\
\hline
\end{tabular}




\begin{tabular}{|c|c|c|c|c|c|c|c|c|}
\hline \multicolumn{2}{|c|}{ Liver metastases } & \multirow[t]{2}{*}{$p$-Value } & \multicolumn{2}{|c|}{ Lung metastases } & \multirow[t]{2}{*}{$p$-Value } & \multicolumn{2}{|c|}{ Distant lymph nodes } & \multirow[t]{2}{*}{$p$-Value } \\
\hline Yes (\%) & No (\%) & & Yes (\%) & No (\%) & & Yes (\%) & No (\%) & \\
\hline $70(25-98)$ & 70 (17-97) & 0.402 & $70(17-98)$ & $70(21-98)$ & 0.047 & $70(2 I-97)$ & 70 (17-98) & 0.001 \\
\hline $105(23.6)$ & $339(76.4)$ & & $165(37.2)$ & $279(62.6)$ & & $182(4 \mid .0)$ & $262(59.0)$ & \\
\hline $139(26.8)$ & $379(73.2)$ & & $190(36.7)$ & $328(63.3)$ & & $169(32.6)$ & $349(67.4)$ & \\
\hline $146(28.3)$ & $370(71.7)$ & & $196(38.0)$ & $320(62.0)$ & & $178(34.5)$ & $338(65.5)$ & \\
\hline $107(27.9)$ & $281(73.2)$ & & $173(45.1)$ & $211(54.9)$ & & $108(28.1)$ & $276(71.9)$ & \\
\hline $97(27.6)$ & $255(72.4)$ & 0.375 & $126(35.8)$ & $226(64.2)$ & $0.4 I I$ & $109(31.0)$ & $243(69.0)$ & 0.324 \\
\hline $88(24.9)$ & $266(75.1)$ & & $145(4 \mid .0)$ & $209(59.0)$ & & $124(35.0)$ & $230(65.0)$ & \\
\hline $106(27.4)$ & $281(72.6)$ & & $162(41.9)$ & $225(58.1)$ & & $123(31.8)$ & $264(68.2)$ & \\
\hline $110(29.3)$ & $266(70.7)$ & & $140(37.2)$ & $236(62.8)$ & & $136(36.2)$ & $240(63.8)$ & \\
\hline $92(23.4)$ & $301(76.6)$ & & I5I (38.4) & $242(61.6)$ & & $145(36.9)$ & $248(63.1)$ & \\
\hline $423(26.6)$ & $1166(73.4)$ & 0.958 & $6 \mathrm{II}(38.5)$ & $978(6 \mid .5)$ & 0.110 & $549(34.6)$ & $1040(65.4)$ & 0.529 \\
\hline $48(25.1)$ & $143(74.9)$ & & $87(45.5)$ & $104(54.5)$ & & $59(30.9)$ & $132(69.1)$ & \\
\hline $21(26.3)$ & $59(73.7)$ & & $25(31.3)$ & 55 (68.7) & & $29(36.3)$ & $51(63.7)$ & \\
\hline I (50.0) & I (50.0) & & I (50.0) & I (50.0) & & $0(0)$ & $2(100 \%)$ & \\
\hline $366(27.4)$ & $970(72.6)$ & 0.162 & $498(37.3)$ & $838(62.7)$ & 0.027 & $460(34.4)$ & $876(65.6)$ & 0.786 \\
\hline $127(24.1)$ & $399(75.9)$ & & $226(43.0)$ & $300(57.0)$ & & 177 (33.7) & $349(66.3)$ & \\
\hline $238(27.0)$ & $645(73.0)$ & 0.717 & $333(37.7)$ & $550(62.3)$ & 0.573 & $328(37.1)$ & 555 (62.9) & 0.039 \\
\hline $237(26.3)$ & $663(73.7)$ & & $36 I(40.1)$ & 539 (59.9) & & $285(31.7)$ & $615(68.3)$ & \\
\hline $18(22.8)$ & $61(77.2)$ & & $30(38.0)$ & $49(62.0)$ & & $24(30.4)$ & $55(69.6)$ & \\
\hline $5(29.4)$ & $12(70.6)$ & 0.003 & $8(47.1)$ & $9(52.9)$ & 0.240 & $2(11.8)$ & I5 (88.2) & 0.254 \\
\hline $16(23.2)$ & $53(76.8)$ & & $34(49.3)$ & $35(50.7)$ & & $23(33.3)$ & $46(66.7)$ & \\
\hline $338(24.5)$ & $104 \mid(75.5)$ & & $535(38.8)$ & $844(6 \mid .2)$ & & $479(34.7)$ & $900(65.3)$ & \\
\hline 134 (33.8) & $263(66.2)$ & & $147(37.0)$ & $250(63.0)$ & & $133(33.5)$ & $264(66.5)$ & \\
\hline $84(27.2)$ & $225(72.8)$ & $<0.001$ & $125(40.5)$ & $184(59.5)$ & 0.910 & $104(33.7)$ & $205(66.3)$ & 0.588 \\
\hline $172(22.5)$ & $593(77.5)$ & & $297(38.8)$ & $468(61.2)$ & & $249(32.5)$ & $516(67.5)$ & \\
\hline $38(24.1)$ & I $20(75.9)$ & & $60(38.0)$ & $98(62.0)$ & & $53(33.5)$ & $105(66.5)$ & \\
\hline $83(25.4)$ & $244(74.6)$ & & $121(37.0)$ & $206(63.0)$ & & $122(37.3)$ & $205(62.7)$ & \\
\hline $116(38.3)$ & I87 (6I.7) & & 121 (39.9) & $182(60.1)$ & & $109(36.0)$ & $194(64.0)$ & \\
\hline $263(27.5)$ & $692(72.5)$ & 0.277 & $399(41.8)$ & $556(58.2)$ & 0.001 & $204(2 \mid .4)$ & 75 I (78.6) & $<0.001$ \\
\hline $54(27.4)$ & $143(72.6)$ & & $76(38.6)$ & $121(61.4)$ & & $87(44.2)$ & $110(55.8)$ & \\
\hline $84(23.8)$ & $269(76.2)$ & & $118(33.4)$ & $235(66.6)$ & & $199(56.4)$ & $154(43.6)$ & \\
\hline $25(20.2)$ & 99 (79.8) & & $32(25.8)$ & $92(74.2)$ & & $86(69.4)$ & $38(30.6)$ & \\
\hline $67(28.8)$ & 166 (7I.2) & & $99(42.5)$ & $134(57.5)$ & & $61(26.2)$ & $172(73.8)$ & \\
\hline $14(25.5)$ & $4 \mid(74.5)$ & 0.600 & $22(40.0)$ & $33(60.0)$ & $<0.001$ & $15(27.3)$ & 40 (72.7) & 0.324 \\
\hline 114 (28.2) & $290(71.8)$ & & $157(38.9)$ & 247 (6I.I) & & $147(36.4)$ & $257(63.6)$ & \\
\hline $104(24.2)$ & $326(75.8)$ & & $203(47.2)$ & $227(52.8)$ & & $136(31.6)$ & $294(68.4)$ & \\
\hline $261(26.8)$ & $712(73.2)$ & & $342(35.1)$ & $631(64.9)$ & & $339(34.8)$ & $634(65.2)$ & \\
\hline $15(12.1)$ & $109(87.9)$ & $<0.001$ & $40(32.3)$ & 84 (67.7) & 0.127 & $54(43.5)$ & $70(56.5)$ & 0.025 \\
\hline $478(27.5)$ & $1260(72.5)$ & & $684(39.4)$ & $1054(60.6)$ & & $583(33.5)$ & $1155(66.5)$ & \\
\hline $14(14.7)$ & 81 (85.3) & 0.008 & $35(36.8)$ & $60(63.2)$ & 0.746 & 45 (47.4) & $50(52.6)$ & 0.007 \\
\hline $479(27.1)$ & $1288(72.9)$ & & $689(39.0)$ & $1078(61.0)$ & & $592(33.5)$ & $1175(66.5)$ & \\
\hline
\end{tabular}




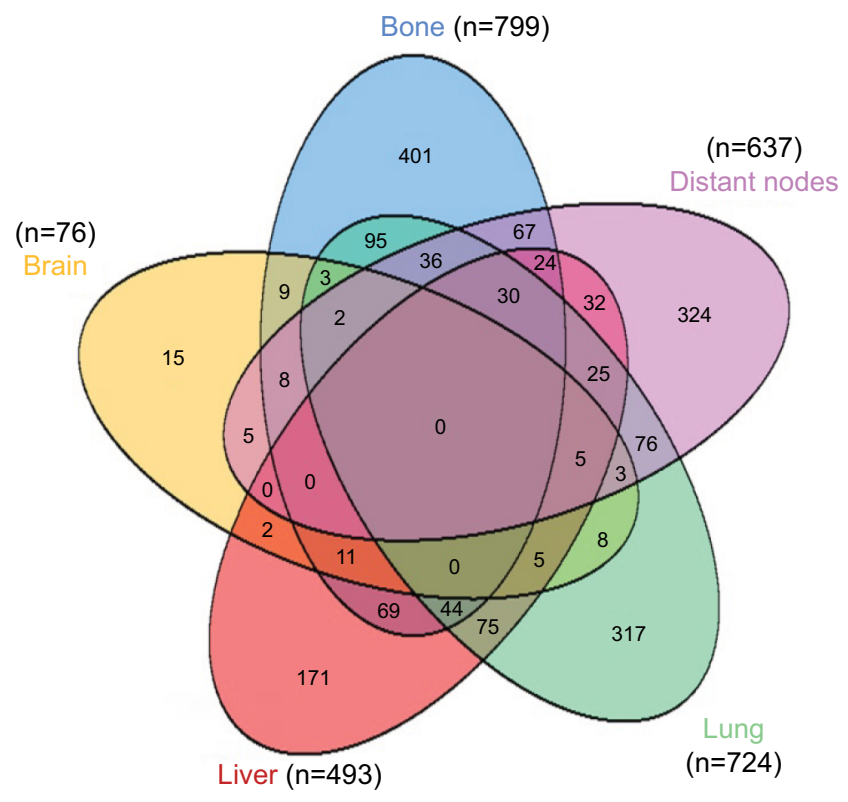

Figure I Venn diagram of the distribution of distant metastatic sites in the overall cohort.

very small number of patients with brain-only metastases (15 patients), they were not included in the Kaplan-Meier analyses shown in Figure 4.

Using multivariate analyses applied to patients with a single metastatic site, bone-only (OS: $\mathrm{HR}=1.562,95 \% \mathrm{CI}=1.115-$ 2.187, $p=0.009$; CSS: HR $=1.169,95 \% \mathrm{CI}=1.169-2.359$, $p=0.005$ ), brain-only (OS: $\mathrm{HR}=3.232,95 \% \mathrm{CI}=1.373-7.612$, $p=0.007$; CSS: $\mathrm{HR}=3.617,95 \% \mathrm{CI}=1.528-8.561, p=0.003$ ), liver-only (OS: $\mathrm{HR}=1.618,95 \% \mathrm{CI}=1.092-2.396, p=0.016$; CSS: HR=1.731, 95\% CI=1.149-2.608, $p=0.009$ ) and lungonly metastases (OS: HR $=1.871,95 \% \mathrm{CI}=1.355-2.584$, $p<0.001$; CSS: $\mathrm{HR}=1.909,95 \% \mathrm{CI}=1.362-2.675, p<0.001$ ) were all found to be adverse prognostic factors for OS and CSS, compared to patients with only distant node metastases (Table 3).

\section{Effect of surgeries to the primary sites} and metastatic sites on survival outcomes

For all patients included in this study, patients who received $\mathrm{RC}$ were associated with better OS $(p<0.001)$ and CSS $(p<0.001)$, regardless of the specific metastatic sites (Figure $5 \mathrm{~A}, \mathrm{~B})$. This phenomenon remained true with regard to patients with a single-site metastasis (Figure 5C, D). In patients with multisite metastases, however, the survival outcomes of patients who received RC did not differ from that of patients with no RC (for OS: $p=0.415$; for CSS: $p=0.649$ ), as shown in Figure 5E, F. The effect of RC on survival outcomes of patients stratified by their metastatic sites was further ana- lyzed and the results revealed that for patients with bone-only (Figure 6A, B), lung-only (Figure 6E, F) and distant node-only metastases (Figure 6G, H), RC could significantly improve the OS and CSS outcomes, while patients with liver metastases (Figure 6C, D) seemed not to benefit from this surgery in terms of OS $(p=0.278)$ and CSS $(p=0.226)$. Multivariate analysis revealed that $\mathrm{RC}$ was a strong prognostic indicator in the overall cohort (OS: $\mathrm{HR}=0.600,95 \% \mathrm{CI}=0.440-0.818$, $p=0.001$; CSS: $\mathrm{HR}=0.632,95 \% \mathrm{CI}=0.462-0.865, p=0.004$; Table 2) and in patients with single metastatic site (OS: $\mathrm{HR}=0.466,95 \% \mathrm{CI}=0.318-0.682, p<0.001 ; \mathrm{CSS}: \mathrm{HR}=0.495$, $95 \%$ CI $=0.336-0.730, p<0.001$; Table 3).

Due to the limitation of the SEER dataset, in which the information of metastasectomy could not be more specific among patients with multiple metastatic sites, the Kaplan-Meier analysis was only carried out in patients with a single-site metastasis when they were stratified by whether the surgeries to the metastatic sites were performed. Figure 7A and $\mathrm{B}$ shows that in all single-metastatic patients, metastasectomy led to a better OS $(p=0.042, \log$-rank test) and single metastatic patients with age $<65$ years had both better OS ( $p=0.006, \log$-rank test) and CSS ( $p=0.014$, logrank test) when metastasectomy was performed (Figure 7C, D). For women patients with single metastatic site, the surgeries to the metastatic sites were associated with better CSS outcomes ( $p=0.049, \log$-rank test), as shown in Figure 7E, F, while MIBC patients seemed to have a better OS after receiving metastasectomy ( $p=0.031$, log-rank test), as shown in Figure 7G, H. Interestingly, statistical differences were found by the Breslow test in some groups of Kaplan-Meier analyses shown in Figure 7. Also, contradictory results were observed between log-rank and Breslow tests in the CSS of all patients with a single metastatic site (log-rank test: $p=0.072$; Breslow test: $p=0.024$; Figure 7B) and of MIBC patients (log-rank test: $p=0.078$; Breslow test: $p=0.016$; Figure $7 \mathrm{H}$ ), and in the OS of women patients with a single metastatic site (log-rank test: $p=0.070$; Breslow test: $p=0.021$; Figure $7 \mathrm{E})$. Nevertheless, after taking all other relevant covariates into consideration, the multivariate analyses did not reveal any prognosis predictive value of metastasectomy, either for the overall cohort (Table 2) or for single-site metastasis patients (Table 3).

\section{Discussion}

Metastatic $\mathrm{BCa}$ was the terminal stage of this malignancy and had rather low survival rates after the diagnosis. Despite a favorable initial response to chemotherapy, long-term OS was achieved by very few patients and the median OS of 

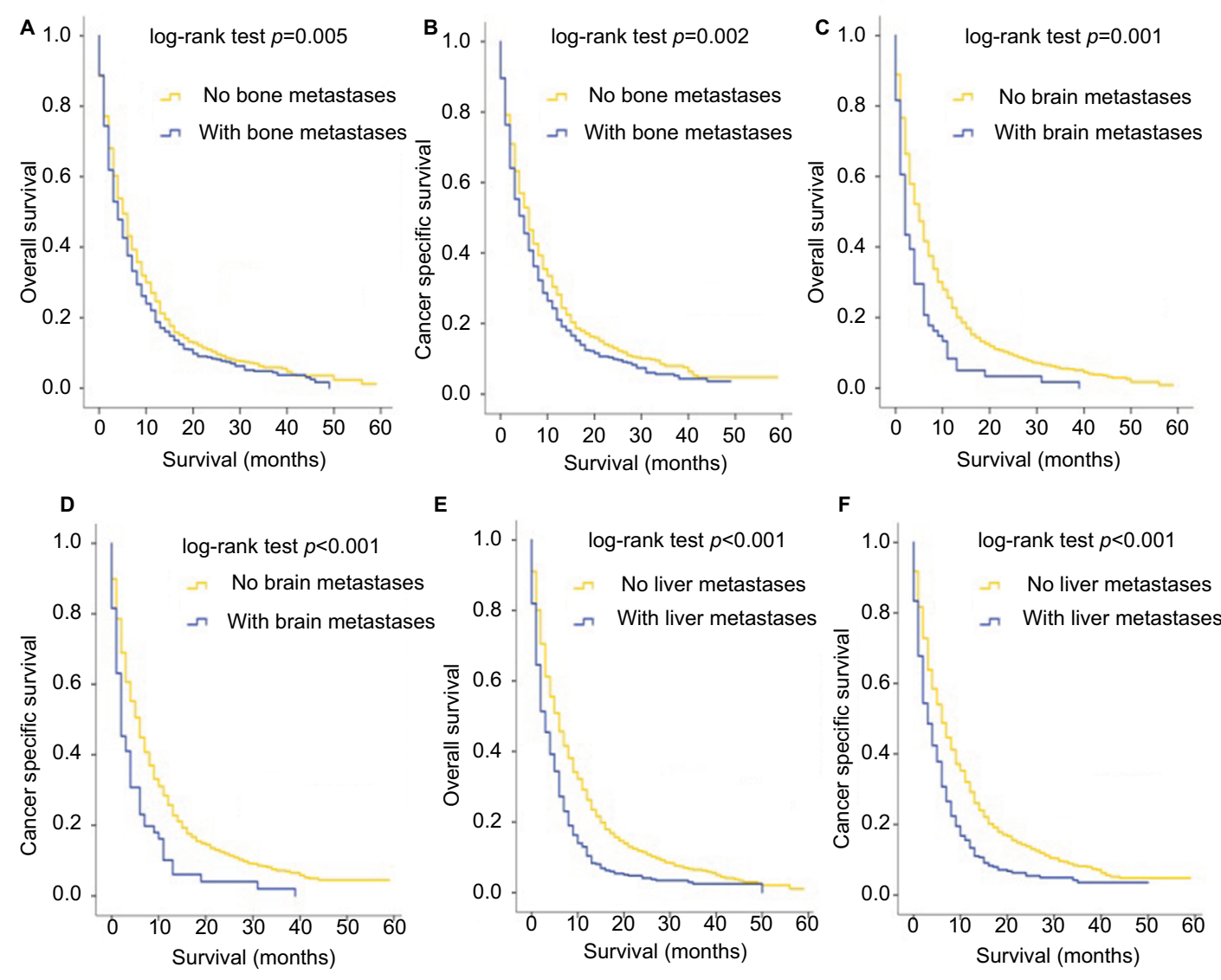

E

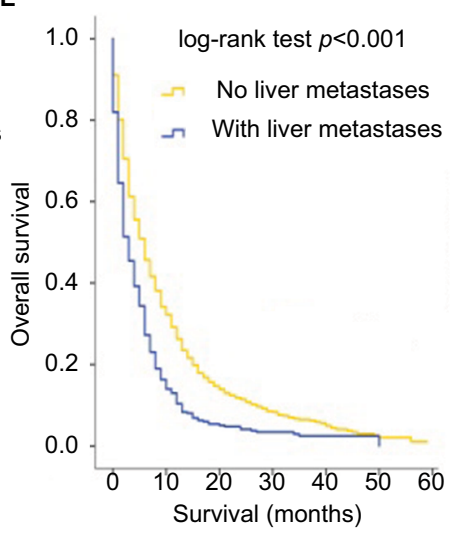

$\mathbf{F}$
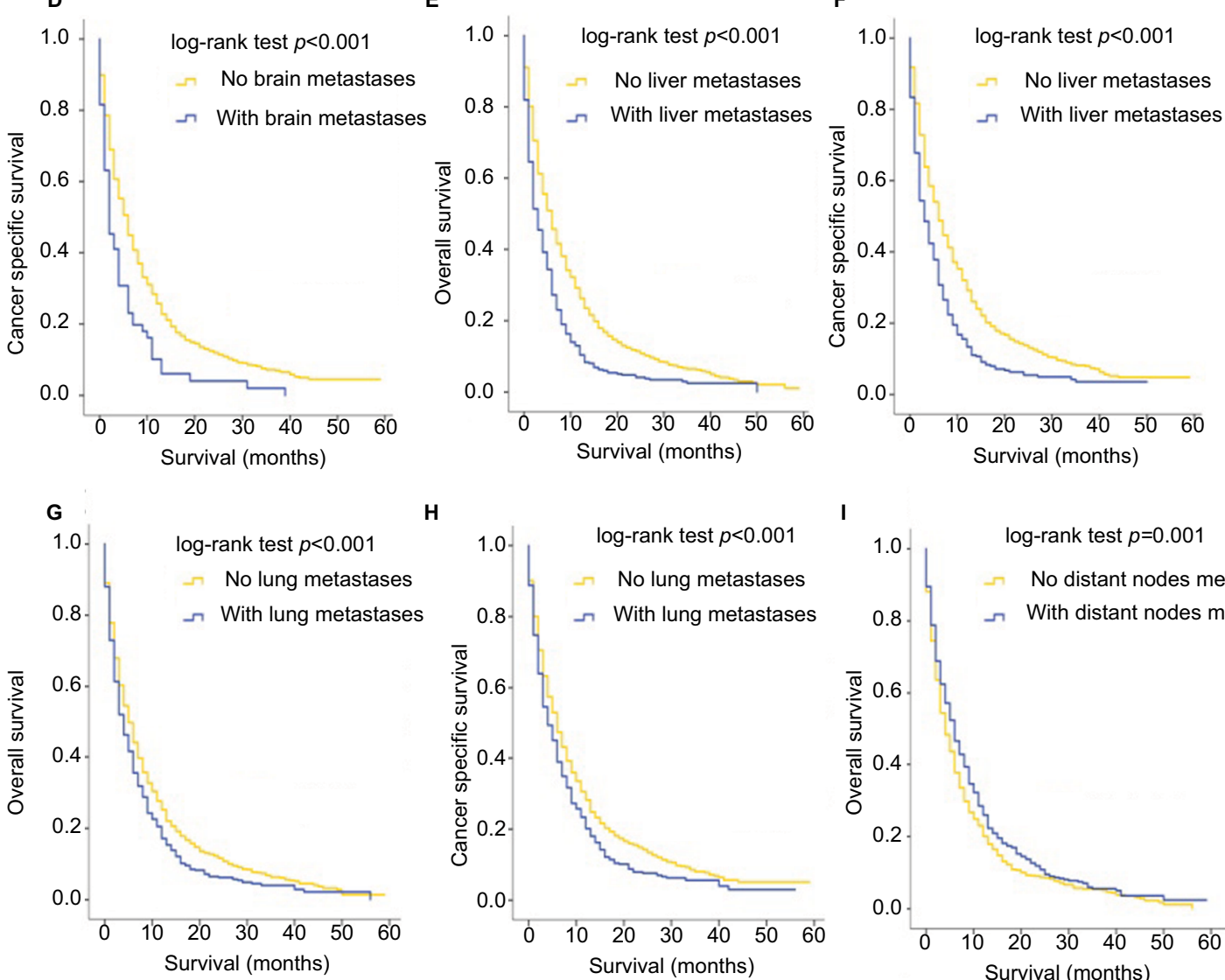

H
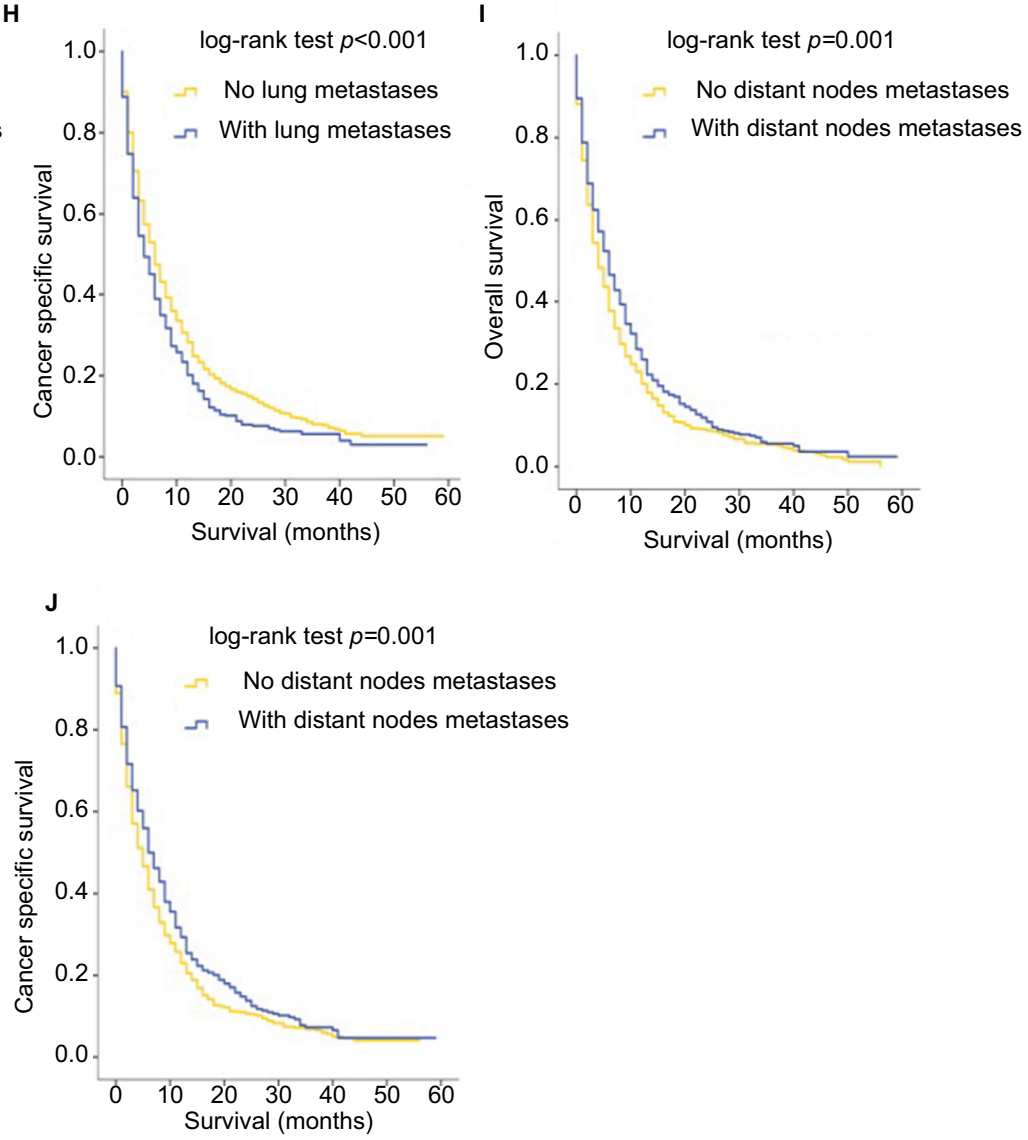

Figure 2 Kaplan-Meier curves of overall survival and cancer-specific survival according to whether or not patients had bone (A, B), brain (C, D), liver (E, F), lung (G, H) and distant lymph node (I, J) metastases. 


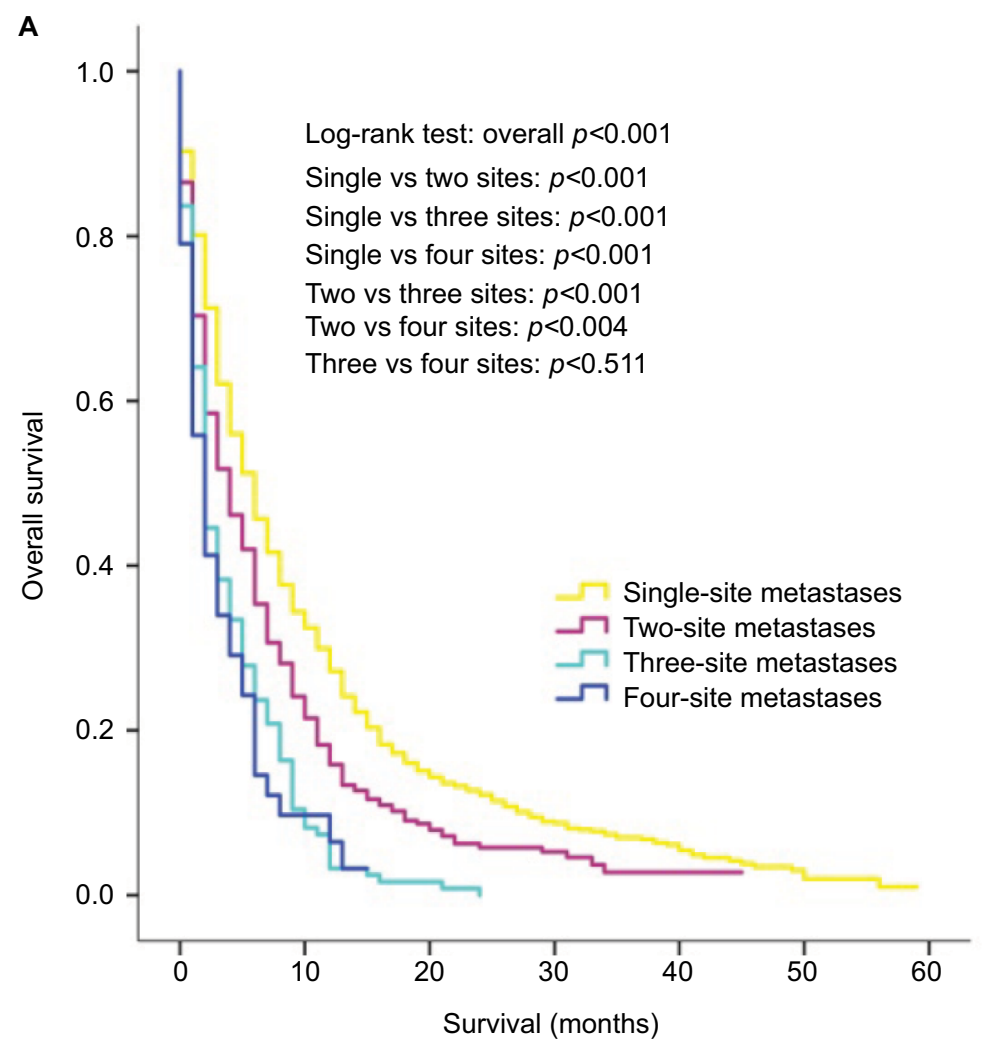

B

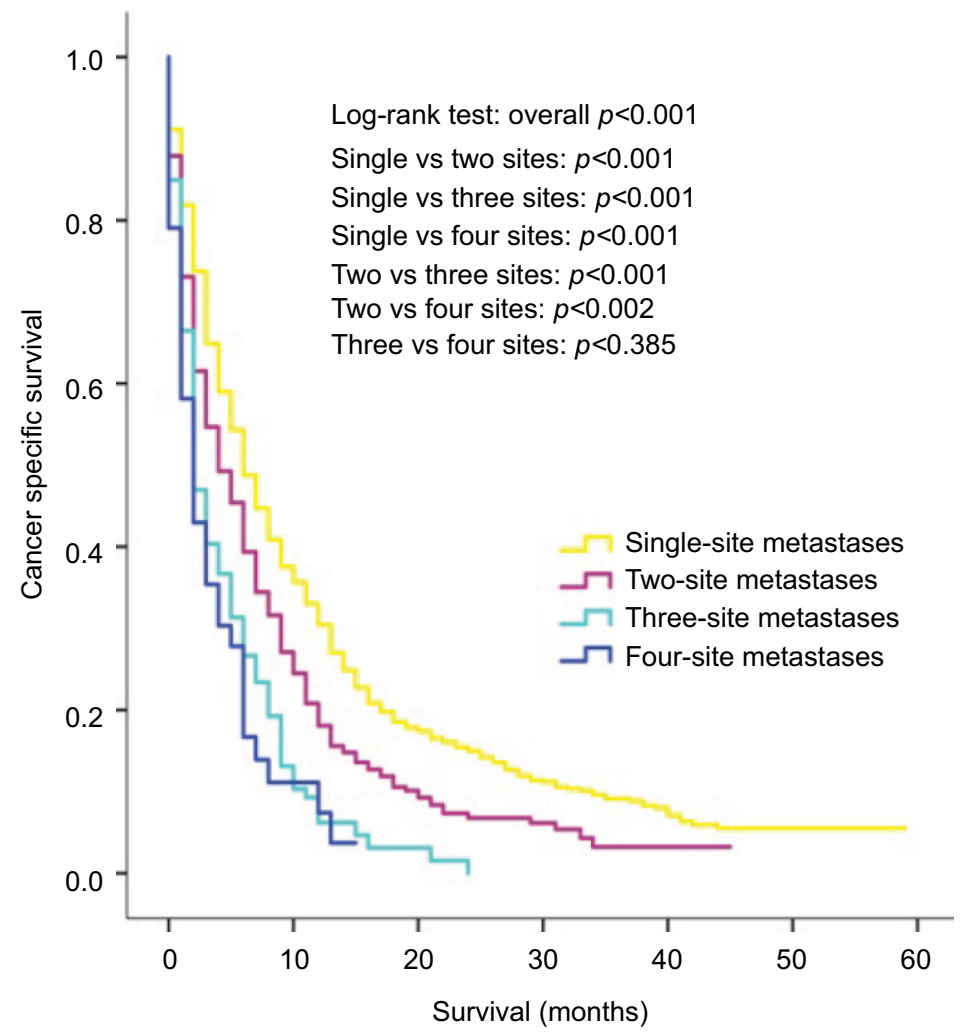

Figure 3 Kaplan-Meier curves of overall survival (A) and cancer-specific survival (B) according to the number of metastatic sites. 
Table 2 Multivariate Cox regression analysis of prognostic factors influencing survival outcomes in overall patient cohort

\begin{tabular}{|c|c|c|c|c|}
\hline \multirow[t]{2}{*}{ Characteristics } & \multicolumn{2}{|l|}{ Overall survival } & \multicolumn{2}{|c|}{ Bladder cancer-specific survival } \\
\hline & Hazard ratio $(95 \% \mathrm{Cl})$ & $p$-Value & Hazard ratio $(95 \% \mathrm{Cl})$ & $p$-Value \\
\hline \multicolumn{5}{|l|}{ Age at diagnosis, years } \\
\hline$\leq 60$ & $0.469(0.353-0.623)$ & $<0.001$ & $0.492(0.366-0.660)$ & $<0.001$ \\
\hline $6 I-70$ & $0.598(0.459-0.78 I)$ & $<0.001$ & $0.594(0.450-0.784)$ & $<0.001$ \\
\hline $7 I-80$ & $0.746(0.576-0.966)$ & 0.026 & $0.765(0.585-1.001)$ & 0.051 \\
\hline$>80$ & Reference & & Reference & \\
\hline \multicolumn{5}{|l|}{ Gender } \\
\hline Male & Reference & & Reference & \\
\hline Female & $0.977(0.799-1.195)$ & 0.821 & $0.989(0.804-1.217)$ & 0.917 \\
\hline \multicolumn{5}{|l|}{ Race } \\
\hline White & Reference & & Reference & \\
\hline Black & $0.957(0.699-1.310)$ & 0.783 & $0.905(0.65 I-1.260)$ & 0.555 \\
\hline Other & I.20I (0.799-I.852) & 0.407 & $1.160(0.738-1.822)$ & 0.521 \\
\hline \multicolumn{5}{|l|}{ Marital status } \\
\hline Unmarried & Reference & 0.311 & Reference & 0.427 \\
\hline Married & $0.909(0.756-1.093)$ & & $0.926(0.765-1.120)$ & \\
\hline \multicolumn{5}{|l|}{ Grade } \\
\hline GI & Reference & & Reference & \\
\hline G2 & $1.800(0.597-5.431)$ & 0.297 & $1.534(0.503-4.683)$ & 0.452 \\
\hline G3 & $1.830(0.666-5.032)$ & 0.242 & $1.715(0.624-4.715)$ & 0.296 \\
\hline \multicolumn{5}{|l|}{ Tumor size, cm } \\
\hline$\leq 2$ & Reference & & Reference & \\
\hline $2-5$ & $1.202(0.805-1.795)$ & 0.369 & I.I44 (0.760-I.722) & 0.518 \\
\hline$>5$ & $1.237(0.83 \mathrm{I}-1.842)$ & 0.294 & I.I 62 (0.775-I.743) & 0.467 \\
\hline \multicolumn{5}{|l|}{ T stage } \\
\hline TI & Reference & & Reference & \\
\hline $\mathrm{T} 2$ & 0.924 (0.7।8-I.189) & 0.540 & $0.925(0.7|2-| .20 \mid)$ & 0.558 \\
\hline T3 & $1.313(0.919-1.875)$ & 0.135 & $1.349(0.936-1.945)$ & 0.109 \\
\hline $\mathrm{T} 4$ & 1.407 (1.022-1.937) & 0.036 & $1.427(1.026-1.984)$ & 0.035 \\
\hline \multicolumn{5}{|l|}{$N$ stage } \\
\hline No & Reference & & Reference & \\
\hline $\mathrm{NI}$ & $1.008(0.753-1.350)$ & 0.959 & I.00I (0.740-I.353) & 0.996 \\
\hline N2 & $1.037(0.8|8-1.3| 4)$ & 0.767 & $1.028(0.805-1.313)$ & 0.823 \\
\hline N3 & I.025 (0.728-I.443) & 0.886 & $0.948(0.66 \mathrm{I}-\mathrm{I} .360)$ & 0.774 \\
\hline \multicolumn{5}{|l|}{ Sites of distant metastases } \\
\hline Bone - yes vs no & $1.679(1.204-2.340)$ & 0.002 & 1.659 (1.172-2.349) & 0.004 \\
\hline Brain - yes vs no & $2.462(I .450-4.18 I)$ & 0.001 & $2.46 \mathrm{I}(\mathrm{I} .428-4.240)$ & 0.001 \\
\hline Liver - yes vs no & $2.038(1.458-2.848)$ & $<0.001$ & 1.935 (1.362-2.747) & $<0.001$ \\
\hline Lung - yes vs no & 1.995 (1.393-2.745) & $<0.001$ & $1.887(1.323-2.693)$ & $<0.001$ \\
\hline Distant nodes - yes vs no & $1.262(0.896-1.777)$ & 0.183 & $1.207(0.844-1.726)$ & 0.304 \\
\hline The number of distant metastases & & & & 0.376 \\
\hline Single-site metastasis & Reference & & Reference & \\
\hline Multisite metastases & $0.777(0.507-1.193)$ & 0.250 & $0.818(0.525-1.276)$ & \\
\hline \multicolumn{5}{|l|}{ Radical cystectomy } \\
\hline No & Reference & & Reference & 0.004 \\
\hline Yes & $0.600(0.440-0.818)$ & 0.001 & $0.632(0.462-0.865)$ & \\
\hline \multicolumn{5}{|l|}{ Metastasectomy } \\
\hline No & Reference & 0.359 & Reference & 0.307 \\
\hline Yes & $1.202(0.81 \mathrm{I}-1.779)$ & & $1.232(0.825-1.840)$ & \\
\hline
\end{tabular}

metastatic BCa typically plateaued at $\sim 14-15$ months. ${ }^{13}$ In 1982, Cowles et al for the first time reported the survival outcomes of six BCa patients with lung metastases and analyzed the function of surgical resection of the solitary pulmonary metastases. ${ }^{17}$ Since then, several studies have focused on the survival outcomes in patients with bone, ${ }^{18,19}$ brain, ${ }^{19-23}$ liver, ${ }^{18}$ lung,,$^{11,17-19,22,24-26}$ distant lymph nodes ${ }^{11,18,19,22,26-28}$ and even skin ${ }^{18,19}$ metastases from BCa. However, few studies focused on the survival differences between patients with different kinds of metastases and no study about $\mathrm{BCa}$ paid atten- 


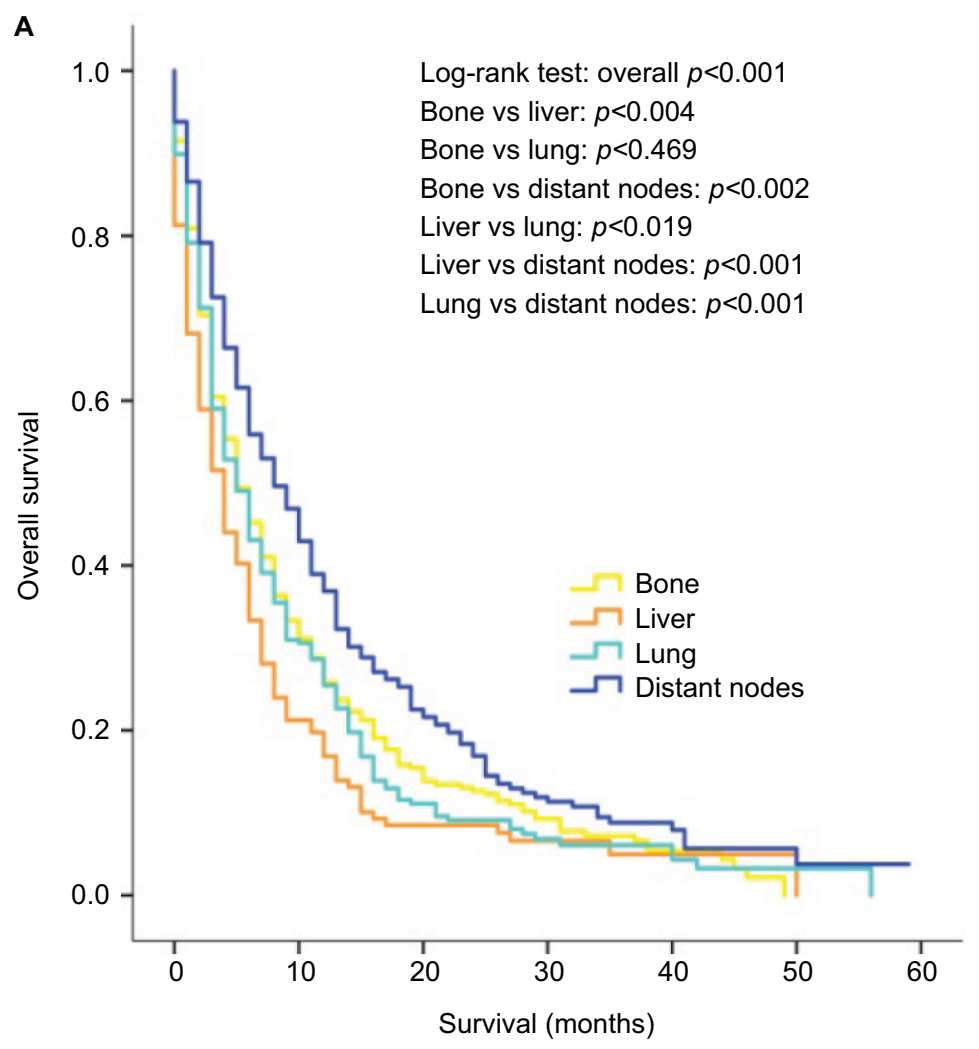

B

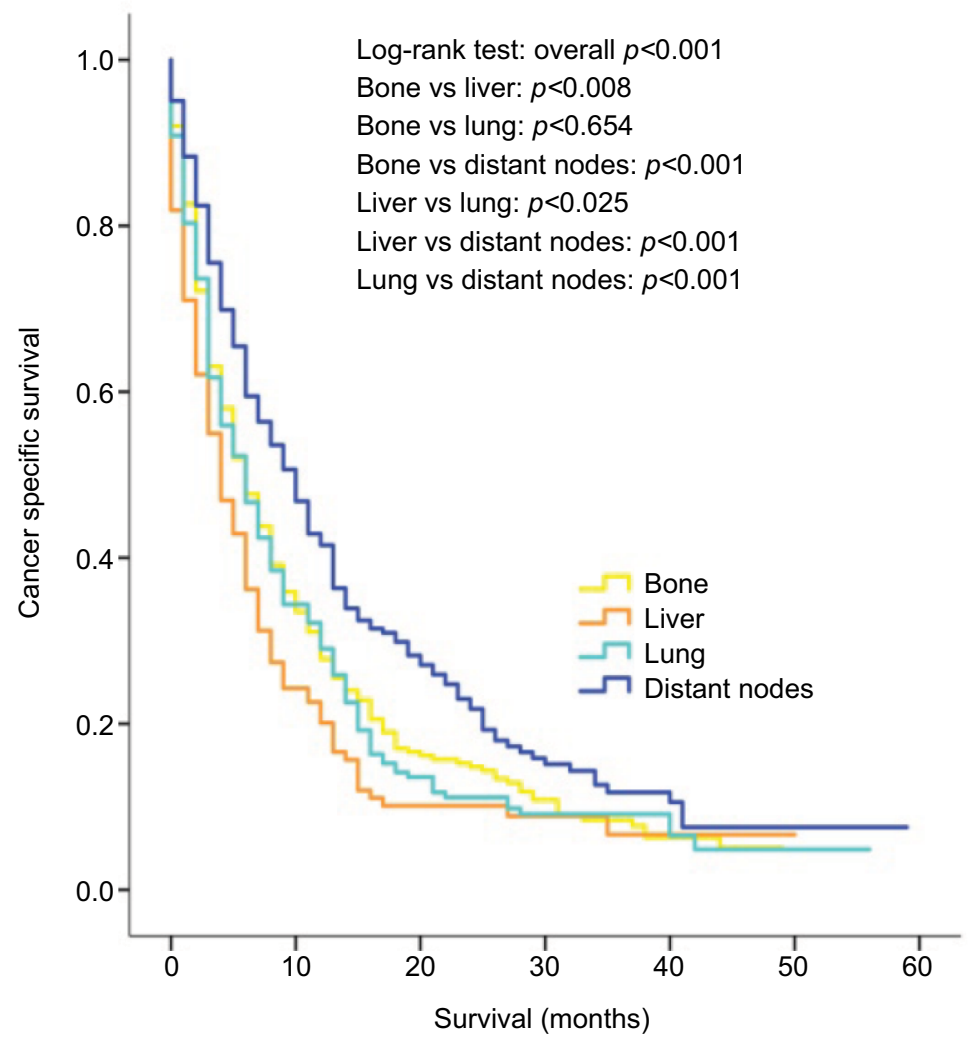

Figure 4 Kaplan-Meier curves of overall survival (A) and cancer-specific survival (B) according to the sites of metastases in patients with single metastatic site. 
Table 3 Multivariate Cox regression analysis of prognostic factors influencing survival outcomes in patients with single metastatic site

\begin{tabular}{|c|c|c|c|c|}
\hline \multirow[t]{2}{*}{ Characteristics } & \multicolumn{2}{|l|}{ Overall survival } & \multicolumn{2}{|c|}{ Bladder cancer-specific survival } \\
\hline & Hazard ratio $(95 \% \mathrm{Cl})$ & $p$-Value & Hazard ratio $(95 \% \mathrm{Cl})$ & $p$-Value \\
\hline \multicolumn{5}{|c|}{ Age at diagnosis, years } \\
\hline$\leq 60$ & $0.464(0.327-0.661)$ & $<0.001$ & $0.495(0.345-0.710)$ & $<0.001$ \\
\hline $6 \mathrm{I}-70$ & $0.597(0.435-0.819)$ & 0.001 & $0.574(0.4 \mid 2-0.799)$ & 0.001 \\
\hline $7 I-80$ & $0.862(0.635-1.170)$ & 0.341 & $0.864(0.628-1.187)$ & 0.366 \\
\hline$>80$ & Reference & & Reference & \\
\hline \multicolumn{5}{|l|}{ Gender } \\
\hline Male & Reference & & Reference & \\
\hline Female & $0.893(0.690-1.156)$ & 0.390 & $0.892(0.683-1.165)$ & 0.402 \\
\hline \multicolumn{5}{|l|}{ Race } \\
\hline White & Reference & & Reference & \\
\hline Black & 1.091 (0.725-1.640) & 0.677 & $1.074(0.705-1.637)$ & 0.740 \\
\hline Other & $1.284(0.754-2.189)$ & 0.358 & $1.306(0.753-2.265)$ & 0.342 \\
\hline \multicolumn{5}{|l|}{ Marital status } \\
\hline Unmarried & Reference & & Reference & \\
\hline Married & $0.842(0.668-1.059)$ & 0.142 & $0.872(0.688-1.107)$ & 0.262 \\
\hline \multicolumn{5}{|l|}{ Grade } \\
\hline $\mathrm{Gl}$ & Reference & & Reference & \\
\hline G2 & $3.626(0.774-17.000)$ & 0.102 & $3.216(0.680-15.208)$ & 0.141 \\
\hline G3 & $2.963(0.708-12.401)$ & 0.137 & $2.695(0.644-11.280)$ & 0.175 \\
\hline \multicolumn{5}{|l|}{ Tumor size, cm } \\
\hline$\leq 2$ & Reference & & Reference & \\
\hline $2-5$ & $1.163(0.730-1.85 \mathrm{I})$ & 0.525 & $1.065(0.666-1.702)$ & 0.794 \\
\hline$>5$ & I.I63 (0.739-I.83I) & 0.515 & $1.070(0.677-1.690)$ & 0.773 \\
\hline \multicolumn{5}{|l|}{ T stage } \\
\hline TI & Reference & & Reference & \\
\hline $\mathrm{T} 2$ & $0.919(0.668-1.266)$ & 0.606 & $0.915(0.658-1.273)$ & 0.599 \\
\hline T3 & $1.655(1.053-2.600)$ & 0.029 & $1.653(1.039-2.631)$ & 0.034 \\
\hline $\mathrm{T} 4$ & $1.723(1.155-2.57 \mathrm{I})$ & 0.008 & $1.687(1.116-2.550)$ & 0.013 \\
\hline \multicolumn{5}{|l|}{$\mathrm{N}$ stage } \\
\hline No & Reference & & Reference & \\
\hline $\mathrm{NI}$ & I.08I (0.739-I.58I) & 0.688 & $1.062(0.7 \mid 5-1.577)$ & 0.767 \\
\hline N2 & $\mathrm{I} .046(0.78 \mathrm{I}-\mathrm{I} .40 \mathrm{I})$ & 0.763 & $1.037(0.767-1.403)$ & 0.811 \\
\hline N3 & $1.178(0.725-1.913)$ & 0.509 & 1.161 (0.700-1.928) & 0.563 \\
\hline \multicolumn{5}{|c|}{ Sites of distant metastases } \\
\hline Bone only & $1.562(1.115-2.187)$ & 0.009 & $1.660(1.169-2.359)$ & 0.005 \\
\hline Brain only & $3.232(1.373-7.612)$ & 0.007 & $3.617(1.528-8.561)$ & 0.003 \\
\hline Liver only & $1.618(1.092-2.396)$ & 0.016 & 1.731 (1.149-2.608) & 0.009 \\
\hline Lung only & I.87I (1.355-2.584) & $<0.001$ & $1.909(1.362-2.675)$ & $<0.001$ \\
\hline Distant nodes only & Reference & & Reference & \\
\hline \multicolumn{5}{|l|}{ Radical cystectomy } \\
\hline No & Reference & & Reference & \\
\hline Yes & $0.466(0.318-0.682)$ & $<0.001$ & $0.495(0.336-0.730)$ & $<0.001$ \\
\hline \multicolumn{5}{|l|}{ Metastasectomy } \\
\hline No & Reference & 0.565 & Reference & 0.514 \\
\hline Yes & I.I57 (0.705-I.898) & & $1.184(0.712-1.969)$ & \\
\hline
\end{tabular}

tion to the important role of the number of metastatic sites and patients with a single metastatic site. Furthermore, the previous studies were all based on small single-institutional patient cohorts (number of bladder patients ranged from 3 to 70$),{ }^{20}$ which obviously restricted the subgroup analyses and stratified studies. Based on the currently largest cohort of metastatic BCa patients, we examined the effect of differ- ent kinds as well as different number of metastatic sites on the prognosis of $\mathrm{BCa}$ patients. In addition, we performed a subgroup analysis focusing on patients with a single metastatic site.

Several of our findings are noteworthy. We found that bone, lung and distant nodes became the top three organs of metastases. This result was not completely in conformity with 

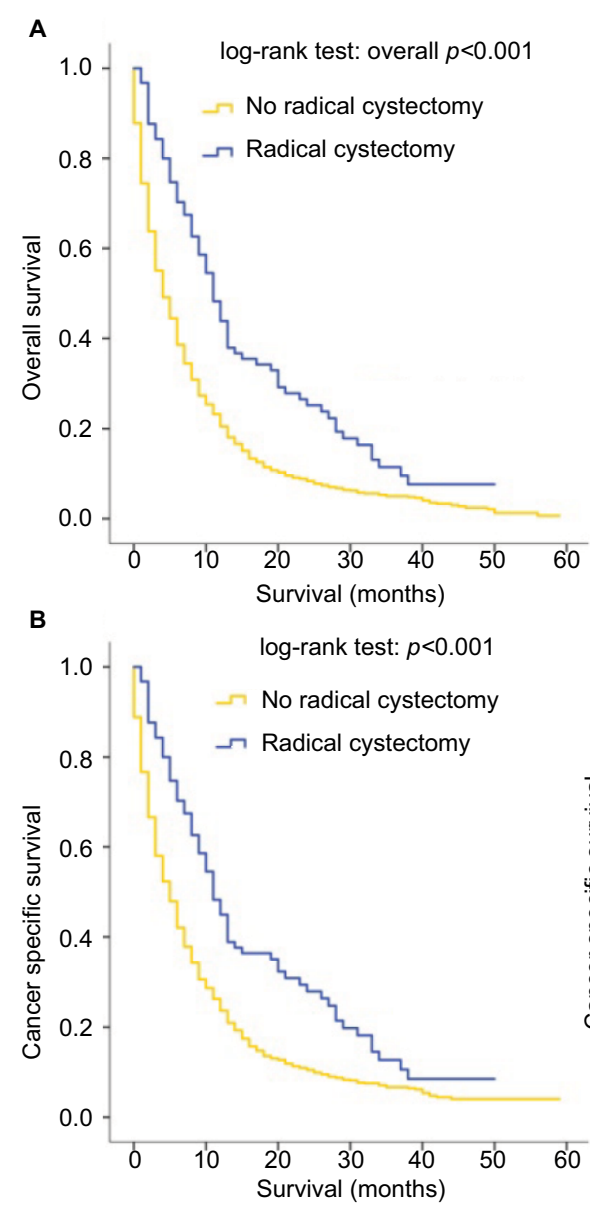
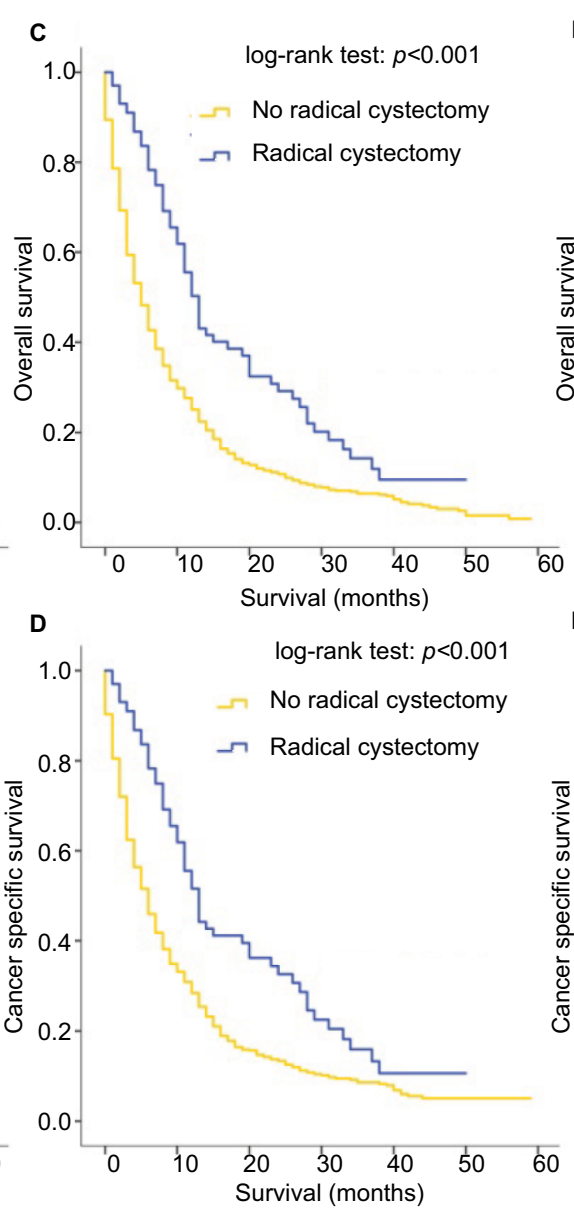

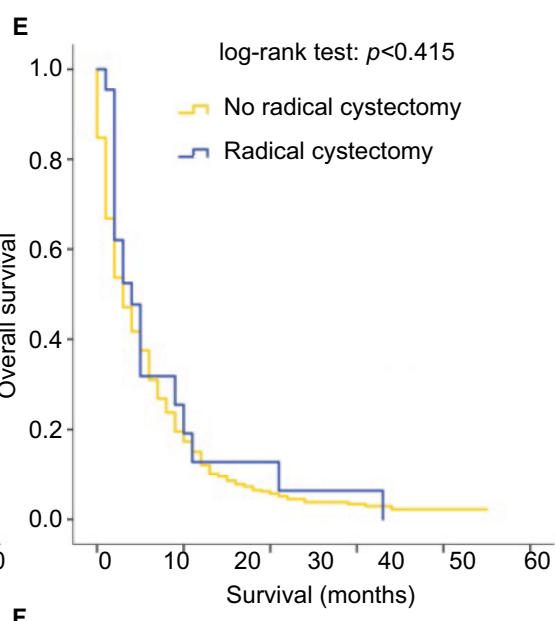

$\mathbf{F}$

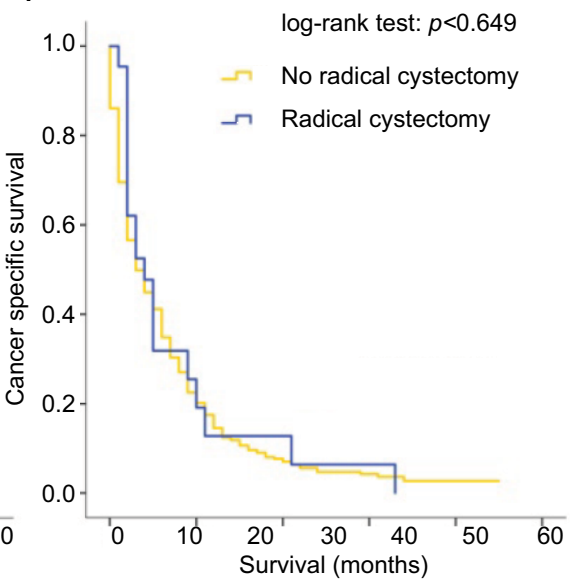

Figure 5 Kaplan-Meier curves of overall survival and cancer-specific survival according to whether or not radical cystectomy has been done for the overall cohort (A, B), in patients with single metastatic site (C, D) and in patients with multiple metastatic sites (E, F).

some of the previous researches. It had been found that lung and distant lymph nodes metastases would always be in the list of the most common sites of metastases of $\mathrm{BCa}$, which was also confirmed in our study. ${ }^{11,18}$ As for bone metastases, Bianchi et al revealed that quite a high bone metastasis rate was also observed in M1 stage BCa patients and this rate was even higher than lung metastasis rate. ${ }^{7}$ Most of the other studies, however, reported a rather small number of patients with bone metastases. Our findings, which were similar to Bianchi's results, showed a higher proportion of bone metastases compared to lung metastases in metastatic BCa patients. This might be due to the different sources of patient information. Bianchi's and our study were both based on national wide patient dataset including enough bone metastases cases, while other studies were all using single-institutional patient cohorts which could lead to the omission of bone metastases. We believed that bone metastases were quite common in patients with $\mathrm{M} 1 \mathrm{BCa}$; therefore, bone scintigraphy might be considered as a routine examination for $\mathrm{BCa}$ patients in order to reduce the missed diagnosis rate of bone metastasis.
The effect of metastatic sites on patients' prognosis had been discussed in several different cancers. ${ }^{29,30}$ For $\mathrm{BCa}$, previous studies have found that liver metastatic sites were independent, unfavorable prognostic factors for OS in patients with advanced urothelial carcinoma. ${ }^{31,32}$ Nakagawa et al suggested the association of bone and liver metastases with poor OS using univariate regression model. ${ }^{33}$ However, the prognostic value of distant metastatic sites in M1 stage BCa still remains unclear. For the first time, we revealed that patients with bone, brain, liver and lung metastases were associated with worse OS and CSS. Moreover, using multivariate Cox regression analysis, bone, brain, liver and lung metastases were proved to be independent adverse prognostic indicators for both OS and CSS. During the time of reviewing relevant literature, we found a regrettable phenomenon that when analyzing the impact of distant metastatic sites on prognosis of BCa patients, most of the previous researches used "visceral metastases" to sum up all kinds of organ metastases including lung, liver and other visceral involvement, without offering separate results of different kinds of metastases. 

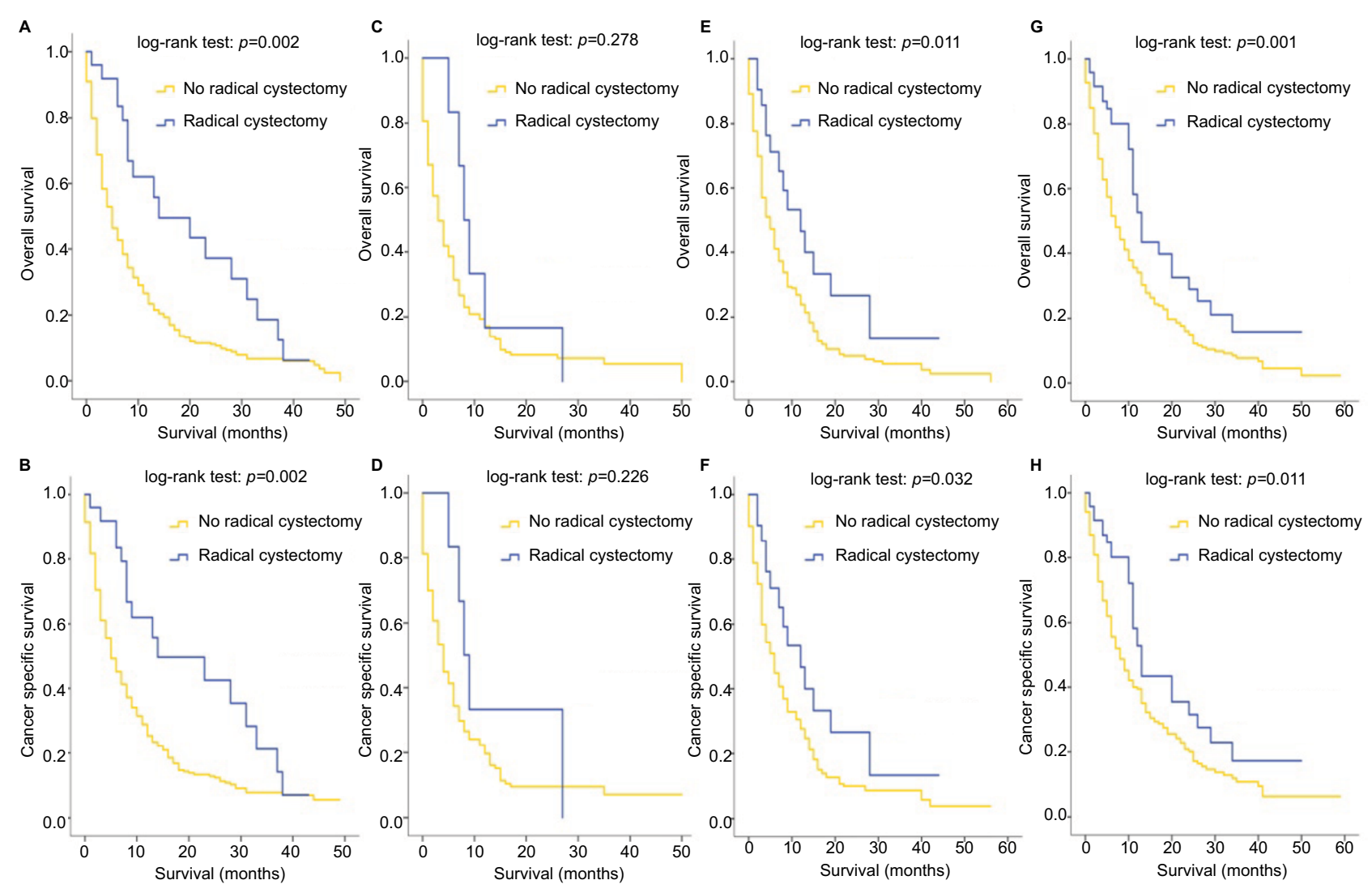

Figure 6 Kaplan-Meier curves of overall survival and cancer-specific survival according to whether or not radical cystectomy has been done for patients with bone-only $(\mathbf{A}, \mathbf{B})$, liver-only (C, D), lung-only (E, F) and distant node-only $(\mathbf{G}, \mathbf{H})$ metastases.

This might be caused by the limited population of patients in those studies. For example, based on a cohort with less than a hundred $\mathrm{BCa}$ patients, Taguchi et al pointed out that patients with visceral metastasis seemed to have prognostic value for OS outcomes, ${ }^{10}$ without analyzing which kind of metastases played roles. ${ }^{10}$ We solved this problem by using a large population of patients and further proved that distant lymph node metastasis was not an independent prognostic factor of metastatic BCa.

In our study, patients with a single metastatic site seemed to be related with better survival compared with those with two, three or four metastatic sites. But in multivariate Cox regression analysis, single-site metastasis was not found to be a significantly independent prognostic predictor of OS and CSS. Our findings were unexpectedly contradictory to the results of the previous study, in which the multisite metastases were found to be able to independently predict worse OS and CSS. ${ }^{9}$ The difference might arise from the different choices of the covariates in the Cox model, and further studies are needed to expound how the number of metastatic sites could affect the prognosis of patients with metastatic $\mathrm{BCa}$. We specially did subgroup analyses based on patients with a single-site metastasis and found that patients with distant node-only and liver-only metastases had the best and the most unfavorable survival outcomes, respectively. The above-mentioned results for the first time focused on single-site metastatic $\mathrm{BCa}$ patients and could contribute to the clinical practice for similar patients.

One of the previous studies had indicated that the surgeries of the primary bladder tumor might contribute to long-term disease free survival in selected patients. ${ }^{34}$ Besides, Chen et al also suggested that the surgeries might improve patients' OS outcomes. ${ }^{9}$ Our study, for the first time, pointed out that patients with single metastatic site rather than multiple metastatic sites could benefit from $\mathrm{RC}$, and that the $\mathrm{RC}$ was an independent prognostic predictor for metastatic BCa patients. Furthermore, this benefit could also be observed in patients with bone-only metastasis. After obtaining such results, it occurred to us that according to recent studies, metastatic prostate cancer patients with oligometastatic sites (low number of nonvisceral metastases) could still benefit from radical prostatectomy. ${ }^{35}$ Was this concept of "oligometastasis" similarly suitable for metastatic $\mathrm{BCa}$ ? What roles does the radical surgery play in patients with metastatic $\mathrm{BCa}$, especially in those with low number of 


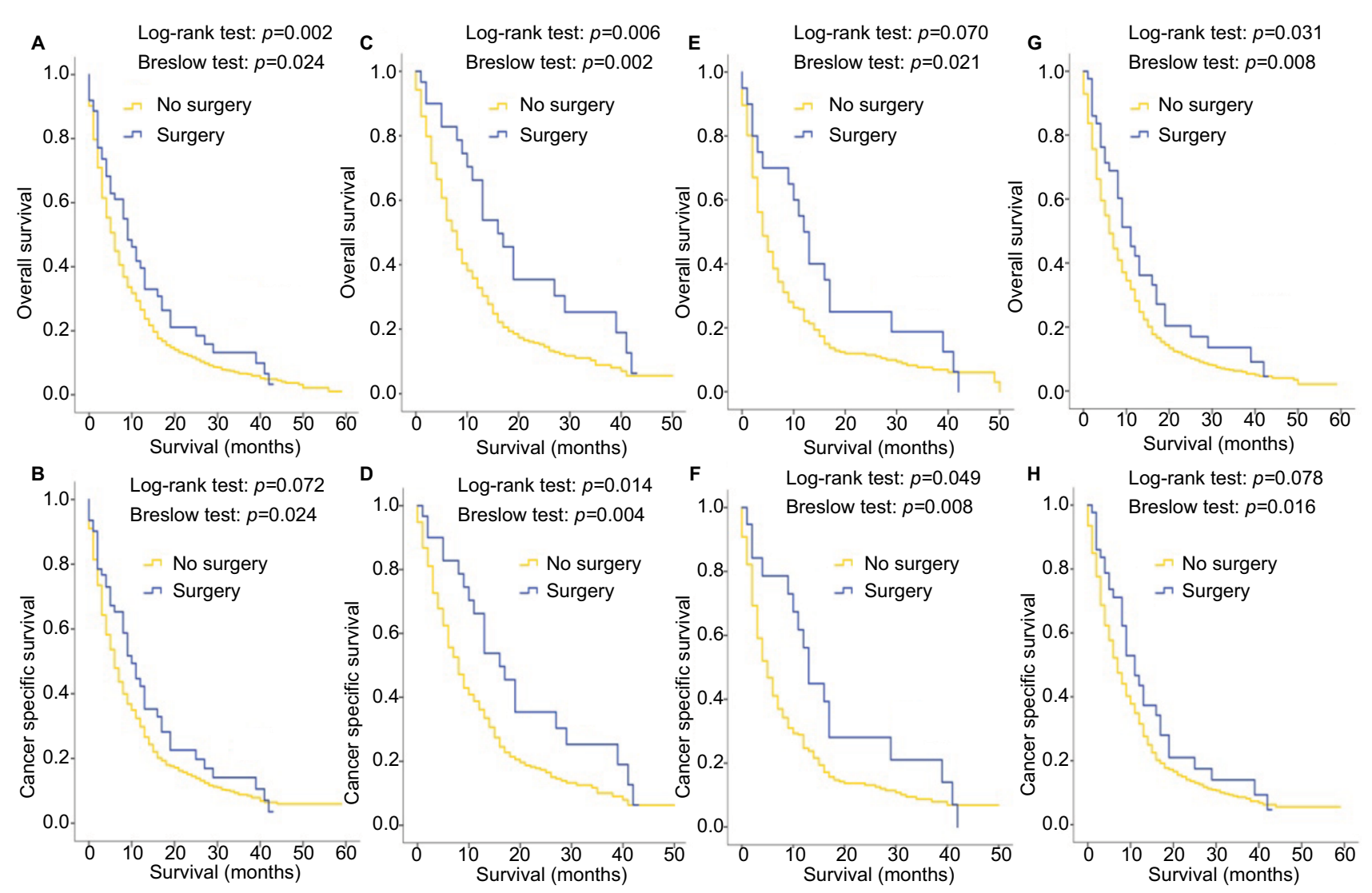

Figure 7 Kaplan-Meier curves of overall survival and cancer-specific survival according to whether or not metastasectomy has been done for all patients with single metastatic site (A, B), single metastatic patients with age $<65$ years (C, D), single metastatic female patients (E, F) and single metastatic patients with muscle-invasive bladder cancer $(\mathbf{G}, \mathbf{H})$.

bone metastases? To answer these two questions, prospective controlled studies are thus needed for the purpose of improving the low survival rates of patients with metastatic BCa. This is especially important, given the insufficiency of relevant studies carried out in the management of this disease.

For a long time, there exists a debate whether surgeries of the metastatic sites, or metastasectomy, could lead to survival benefits for patients with metastatic BCa. A study carried out by Matsuguma et al emphasized that pulmonary metastasectomy might have a curative role in the treatment of BCa with lung metastases. ${ }^{16}$ Similarly, Lehmann et al thought a long-term cancer control and possible cure can be achieved in a subgroup of patients following surgical removal of the metastases. ${ }^{19} \mathrm{~A}$ recently published meta-analysis pointed out that although pooled analyses of studies showed an improved OS for patients treated with metastasectomy compared with nonsurgical treatment of metastatic lesions ( $\mathrm{HR}=0.63 ; 95 \% \mathrm{CI}=0.49-0.81$ ), limited conclusions could be drawn due to lack of uniform reporting elements and multiple sources of bias. ${ }^{8}$ The results of our study were in line with the above-mentioned meta-analysis. Also, we further showed that among patients with a single metastatic site, younger patients, female patients and patients with MIBC seemed to be more suitable for metastasectomy. Interestingly, in this study, we found a statistically improved CSS or OS following metastasectomy in some patient groups using the Breslow test rather than the log-rank test. This phenomenon gave more emphasis to early deaths occurring in patients without receiving metastasectomy. When lengthening the follow-up period, the survival curves became closer or even crossed. This could be the reason why differences existed between log-rank and Breslow tests in our study, since the Breslow test better reflects initial results while the log-rank test more accurately characterizes final outcomes. ${ }^{36}$

Although to the best of our knowledge, this is the largest study evaluating the prognostic significance of the sitespecific metastases of $\mathrm{BCa}$ and the effect of surgeries on patients' prognosis, some potential limitations should still be considered. The major limitation came from the SEER dataset itself. For example, the SEER dataset only included five specific sites of metastases and for multisite metastatic patients, the information of metastasectomy could not be specific to the exact kind of metastatic sites. In addition, there was a lack of details concerning chemotherapy, endocrine 
therapy and targeted treatment. Besides, there might be some bias difficult to avoid due to the nature of respective studies.

\section{Conclusion}

This population-based study demonstrated that distant metastatic sites showed differential effects on survival outcomes in patients with metastatic $\mathrm{BCa}$, and surgeries, including $\mathrm{RC}$ and metastasectomy, might still lead to survival benefits for patients. Further studies are necessary before we establish the selection criteria for patients who may potentially benefit from local treatment of the primary tumor and metastatic diseases.

\section{Acknowledgments}

This study was supported by the National Natural Science Foundation of China (No. 81472379) and the Leading Talent Project of Shanghai.

\section{Disclosure}

The authors report no conflicts of interest in this work.

\section{References}

1. Dong F, Xu T, Shen Y, et al. Dysregulation of miRNAs in bladder cancer: altered expression with aberrant biogenesis procedure. Oncotarget. 2017;8(16):27547-27568.

2. Siegel RL, Miller KD, Jemal A. Cancer statistics, 2016. CA Cancer J Clin. 2016;66(1):7-30.

3. Ferlay J, Soerjomataram I, Dikshit R, et al. Cancer incidence and mortality worldwide: sources, methods and major patterns in GLOBOCAN 2012. Int J Cancer. 2015;136(5):E359-E386.

4. Pruthi RS, Baldwin N, Bhalani V, Wallen EM. Conservative management of low risk superficial bladder tumors. J Urol. 2008;179(1):87-90; discussion 90 .

5. Witjes JA, Compérat E, Cowan NC, et al. Muscle-invasive and metastatic bladder cancer. In: EAU-ESTRO-ESUR-SIOG Guidelines. ed. Arnhem: EAU Guidelines Office; 2017:9-10.

6. Rosenberg JE, Carroll PR, Small EJ. Update on chemotherapy for advanced bladder cancer. J Urol. 2005;174(1):14-20.

7. Bianchi M, Roghmann F, Becker A, et al. Age-stratified distribution of metastatic sites in bladder cancer: a population-based analysis. Can Urol Assoc J. 2014;8(3-4):E148-E158.

8. Patel V, Collazo Lorduy A, Stern A, et al. Survival after metastasectomy for metastatic urothelial carcinoma: a systematic review and metaanalysis. Bladder Cancer. 2017;3(2):121-132.

9. Chen C, Hu L, Chen Y, Hou J. The prognostic value of histological subtype in patients with metastatic bladder cancer. Oncotarget. 2017;8(17):28408-28417.

10. Taguchi S, Nakagawa T, Uemura Y, et al. Validation of major prognostic models for metastatic urothelial carcinoma using a multi-institutional cohort of the real world. World J Urol. 2016;34(2):163-171.

11. Abe T, Kitamura H, Obara W, et al. Outcome of metastasectomy for urothelial carcinoma: a multi-institutional retrospective study in Japan. J Urol. 2014;191(4):932-936.

12. Pond GR, Agarwal N, Bellmunt J, et al. A nomogram including baseline prognostic factors to estimate the activity of second-line therapy for advanced urothelial carcinoma. BJU Int. 2014;113(5b):E137-E143.

13. von der Maase H, Sengelov L, Roberts JT, et al. Long-term survival results of a randomized trial comparing gemcitabine plus cisplatin, with methotrexate, vinblastine, doxorubicin, plus cisplatin in patients with bladder cancer. J Clin Oncol. 2005;23(21):4602-4608.
14. Sternberg CN, Vogelzang NJ. Gemcitabine, paclitaxel, pemetrexed and other newer agents in urothelial and kidney cancers. Crit Rev Oncol Hematol. 2003;46(Suppl):S105-S115.

15. Kim T, Ahn JH, You D, et al. Pulmonary metastasectomy could prolong overall survival in select cases of metastatic urinary tract cancer. Clin Genitourin Cancer. 2015;13(4):e297-e304.

16. Matsuguma $\mathrm{H}$, Yoshino $\mathrm{I}$, Ito $\mathrm{H}$, et al. Is there a role for pulmonary metastasectomy with a curative intent in patients with metastatic urinary transitional cell carcinoma? Ann Thorac Surg. 2011;92(2): 449-453.

17. Cowles RS, Johnson DE, McMurtrey MJ. Long-term results following thoracotomy for metastatic bladder cancer. Urology. 1982;20(4):390-392.

18. Otto T, Krege S, Suhr J, Rubben H. Impact of surgical resection of bladder cancer metastases refractory to systemic therapy on performance score: a phase II trial. Urology. 2001;57(1):55-59.

19. Lehmann J, Suttmann H, Albers P, et al. Surgery for metastatic urothelial carcinoma with curative intent: the German experience (AUO AB 30/05). Eur Urol. 2009;55(6):1293-1299.

20. Anderson RS, el-Mahdi AM, Kuban DA, Higgins EM. Brain metastases from transitional cell carcinoma of urinary bladder. Urology. 1992;39(1):17-20.

21. Mahmoud-Ahmed AS, Suh JH, Kupelian PA, et al. Brain metastases from bladder carcinoma: presentation, treatment and survival. J Urol. 2002;167(6):2419-2422.

22. Siefker-Radtke AO, Walsh GL, Pisters LL, et al. Is there a role for surgery in the management of metastatic urothelial cancer? The M. D. Anderson experience. J Urol. 2004;171(1):145-148.

23. Fokas E, Henzel M, Engenhart-Cabillic R. A comparison of radiotherapy with radiotherapy plus surgery for brain metastases from urinary bladder cancer: analysis of 62 patients. Strahlenther Onkol. 2010;186(10):565-571.

24. Kanzaki R, Higashiyama M, Fujiwara A, et al. Outcome of surgical resection of pulmonary metastasis from urinary tract transitional cell carcinoma. Interact Cardiovasc Thorac Surg. 2010;11(1):60-64.

25. Matsuguma H, Yoshino I, Ito H, et al; Metastatic Lung Tumor Study Group of Japan. Is there a role for pulmonary metastasectomy with a curative intent in patients with metastatic urinary transitional cell carcinoma? Ann Thorac Surg. 2011;92(2):449-453.

26. Bekku K, Saika T, Kobayashi Y, et al. Could salvage surgery after chemotherapy have clinical impact on cancer survival of patients with metastatic urothelial carcinoma? Int J Clin Oncol. 2013;18(1): $110-115$.

27. Sweeney P, Millikan R, Donat M, et al. Is there a therapeutic role for post-chemotherapy retroperitoneal lymph node dissection in metastatic transitional cell carcinoma of the bladder? J Urol. 2003;169(6): 2113-2117.

28. Necchi A, Giannatempo P, Lo Vullo S, et al. Postchemotherapy lymphadenectomy in patients with metastatic urothelial carcinoma: long-term efficacy and implications for trial design. Clin Genitourin Cancer. 2015;13(1):80-86.e1.

29. Wu SG, Li H, Tang LY, et al. The effect of distant metastases sites on survival in de novo stage-IV breast cancer: a SEER database analysis. Tumour Biol. 2017;39(6):1010428317705082.

30. Oweira H, Petrausch U, Helbling D, et al. Prognostic value of sitespecific metastases in pancreatic adenocarcinoma: a surveillance epidemiology and end results database analysis. World J Gastroenterol. 2017;23(10):1872-1880.

31. Bellmunt J, Choueiri TK, Fougeray R, et al. Prognostic factors in patients with advanced transitional cell carcinoma of the urothelial tract experiencing treatment failure with platinum-containing regimens. $J$ Clin Oncol. 2010;28(11):1850-1855.

32. Sonpavde G, Pond GR, Fougeray R, et al. Time from prior chemotherapy enhances prognostic risk grouping in the second-line setting of advanced urothelial carcinoma: a retrospective analysis of pooled, prospective phase 2 trials. Eur Urol. 2013;63(4):717-723.

33. Nakagawa T, Hara T, Kawahara T, et al. Prognostic risk stratification of patients with urothelial carcinoma of the bladder with recurrence after radical cystectomy. J Urol. 2013;189(4):1275-1281. 
34. Herr HW, Donat SM, Bajorin DF. Post-chemotherapy surgery in patients with unresectable or regionally metastatic bladder cancer. $J$ Urol. 2001;165(3):811-814.

35. Gandaglia G, Fossati N, Stabile A, et al. Radical prostatectomy in men with oligometastatic prostate cancer: results of a single-institution series with long-term follow-up. Eur Urol. 2017;72(2):289-292.
36. Santambrogio R, Opocher E, Costa M, Bruno S, Ceretti AP, Spina GP. Natural history of a randomized trial comparing distal spleno-renal shunt with endoscopic sclerotherapy in the prevention of variceal rebleeding: a lesson from the past. World J Gastroenterol. 2006;12(39): 6331-6338.

\section{Publish your work in this journal}

Cancer Management and Research is an international, peer-reviewed open access journal focusing on cancer research and the optimal use of preventative and integrated treatment interventions to achieve improved outcomes, enhanced survival and quality of life for the cancer patient. The manuscript management system is completely online and includes a very quick and fair peer-review system, which is all easy to use. Visit http://www.dovepress.com/testimonials.php to read real quotes from published authors. 